\title{
TTR
}

Traduction, terminologie, re?daction

\section{Lavoie, Judith. Mark Twain et la parole noire, Montréal, Les Presses de l’Université de Montréal, 2002, 221 p.}

\section{Savoyane Henri-Lepage}

Volume 16, numéro 1, 1er semestre 2003

Controverse en traductologie

Controversy in Translation Studies

URI : https://id.erudit.org/iderudit/008564ar

DOI : https://doi.org/10.7202/008564ar

Aller au sommaire du numéro

\section{Éditeur(s)}

Association canadienne de traductologie

ISSN

0835-8443 (imprimé)

1708-2188 (numérique)

Découvrir la revue

Citer ce compte rendu

Henri-Lepage, S. (2003). Compte rendu de [Lavoie, Judith. Mark Twain et la parole noire, Montréal, Les Presses de l'Université de Montréal, 2002, 221 p.] TTR, 16(1), 243-246. https://doi.org/10.7202/008564ar d'utilisation que vous pouvez consulter en ligne.

https://apropos.erudit.org/fr/usagers/politique-dutilisation/ 


\section{COMPTES RENDUS}

\section{Lavoie, Judith. Mark Twain et la parole noire, Montréal, Les Presses de l’Université de Montréal, 2002, 221 p.}

Dans cet ouvrage qui a obtenu le Prix du Gouverneur Général en 2002, Judith Lavoie se penche sur les multiples traductions françaises du roman de Mark Twain, Adventures of Huckleberry Finn, publié aux États-Unis en 1885. La première traduction, par William-Little Hughes, sera publiée un an plus tard en France. Il faudra ensuite attendre 1948 pour lire la première retraduction, par Suzanne Nétillard, qui sera suivie de six retraductions. Dans sa thèse de doctorat, Lavoie étudiait toutes les traductions françaises, mais dans cet ouvrage, elle se concentre sur les traductions de Hughes et de Nétillard, tout en dressant de brefs parallèles avec celles de Yolande et René Surleau (1950), d’André Bay (1961), de Lucienne Molitor (1963), de Jean La Gravière (1979), et enfin de Claire Laury (1979). La tâche de Lavoie sera ici de dégager les projets de traduction, qu'elle définit comme un système de choix de traduction actualisés dans le texte, de Hughes et de Nétillard respectivement, après avoir mis au jour les visées mêmes de Twain dans le roman.

Le premier des quatre chapitres qui composent cet ouvrage s'intitule "Adventures of Huckleberry Finn: l'ironie comme outil dénonciateur » et propose une lecture du roman de Twain. Pour Lavoie, la principale visée de Twain serait de "dénoncer ou remettre en question la société blanche (esclavagiste) du Sud des États-Unis de la période pré- et postguerre de Sécession » (p. 19) et son outil de prédilection pour y parvenir résiderait dans l'ironie. La représentation particulière de la parole du personnage de Jim - l'esclave de Miss Watson en fuite avec Huck - est un procédé contribuant à la dénonciation du système esclavagiste. Peu de critiques ayant traité de la parole de Jim, Judith Lavoie tente d'en faire ressortir la double visée : donner un statut littéraire à la parole noire et revendiquer l'humanité de Jim. En effet, son étude met au jour le caractère complexe, multidimensionnel et autonome de Jim en insistant toujours sur son humanité.

Le deuxième chapitre, le plus substantiel, s'intitule "William-Little Hughes et Huckleberry Finn: traduire pour 
aseptiser ». Grâce à un choix judicieux d'exemples, Lavoie démontre que le projet de traduction de Hughes dénote une position idéologique contraire à celle de Twain: "là où l'anglais déstabilisait les idées reçues, le texte français les maintient et les renforce. » (p. 61) Ce nouveau texte résulte d'une série de transformations qui touchent à la narration, à la description et à la composante dialogale. C'est surtout à travers les transformations que Hughes fait subir au personnage de Jim, autant à sa parole, à ses actions qu'à la description qu'en fait le narrateur, que la visée du texte se trouve renversée. En effet, Hughes, au moyen d'ajouts, d'omissions et de déplacements, transforme Jim en un personnage unidimensionnel, soumis, paresseux et incapable de complexité émotive ou d'autonomie, alors que Huck devient un bon petit garçon qui se comporte de façon autoritaire et paternaliste avec Jim.

En somme, Lavoie démontre clairement comment le système de choix opéré par William-Little Hughes dans sa traduction contribue à effacer l'humanité de Jim. De plus, les passages traitant de Miss Watson et pap Finn, représentants de la société blanche dans le roman, subissent des transformations significatives. Là où ils étaient dépeints comme hypocrites et violents dans le texte anglais, ils deviennent inoffensifs, voire gentils et moraux dans la traduction. Pour Lavoie, tous ces déplacements sont loin d'être le fruit du hasard, ils forment plutôt un réseau organisé de choix esthétiques et idéologiques : "des morceaux signifiants tombent en place pour former une réelle mosaïque traductive, c'est-à-dire un ensemble construit et cohérent de choix de traduction. » (p. 141) Les choix de traduction des Surleau, en comparaison, sont subtils, mais atténuent tout de même certains traits de la caractérisation de Jim. La brève adaptation de Laury, quant à elle, est extrémiste; elle va encore plus loin que Hughes dans le renversement de la visée de l'œuvre de départ en transformant Jim en serviteur bête qui, en tant que "nègre d’Amérique », ne prononce par les « $\mathrm{r} »$.

Le troisième chapitre, « Suzanne Nétillard et Huckleberry Finn : un début d'oralité », analyse la première retraduction du roman. Le projet de Nétillard est diamétralement opposé à celui de Hughes, comme en témoignent le discours de Jim, ses actions, ainsi que la préface signée par l'historien marxiste Jean Kanapa. Lavoie, grâce à la confrontation de passages-clés, mais aussi à l'analyse du paratexte, démontre comment Nétillard réactive et accentue même 
parfois le projet du texte de départ. Cette retraduction est par ailleurs la première destinée à un public adulte, ce qui la place à un autre niveau que les traductions étudiées précédemment. Ce changement de public-cible expliquerait donc en partie qu'on ne trouve pas de transformations majeures dans le texte de l'ordre de celles de Hughes ou encore de Laury. La brève mention que fait Lavoie du public jeunesse auquel s'adressait la première traduction mériterait d'être explicitée. Cet élément ne devrait-il pas être pris en compte dans l'analyse comparative des diverses traductions ? Non pas que le public jeunesse puisse justifier un message raciste, mais il pourrait certainement expliquer une certaine simplification de l'action et de la caractérisation des personnages, pour en faire des «bons et des méchants », entraînant ainsi un aplatissement de la complexité du personnage de Jim.

Lavoie remarque avec justesse que Nétillard est la première à marquer le parler de Jim de façon à ce que le lecteur puisse l'identifier aisément. Jim dispose enfin d'un espace langagier propre en français, ce qui lui confère une autonomie discursive, une efficacité rhétorique et une intensité dramatique. Sa parole traduite se trouve quelque part entre le parler paysan et familier: "en plus de témoigner d'une recherche linguistique évidente, le sociolecte traduit de Jim est également le lieu d'une recherche, stylistique cette fois. » (p. 155) Cette recherche n'est pas sans nous rappeler celle de Twain, qui confère, peut-être pour la première fois dans la littérature américaine, un véritable statut littéraire au Black English. Certains mots, expressions et tournures utilisés par Nétillard sont empruntés au français créolisé et font état d'une tentative de recours à ce que Lavoie nomme la « créolisation linguistique ». Enfin, Nétillard fait un réel travail de création et ne vise en aucun cas à reproduire un sociolecte existant, mais plutôt à marquer le parler noir dans la traduction française. L'unité stylistico-sémantique des choix de traduction de Nétillard produit une traduction efficace qui maintient les visées originales : «la version française de Huckleberry Finn par Nétillard est une œuvre plurilingue et plurivoque : divers sociolectes s'y côtoient, diverses valeurs s'y confrontent. » (p. 189) De plus, Lavoie aborde brièvement, dans ce chapitre, les traductions de Bay et de Molitor, qui, à l'instar de celle de Nétillard, témoignent toutes deux d'un désir de réhabilitation du personnage de Jim et de son parler.

Le chapitre final de l'ouvrage, intitulé « Pour la création d'une parole noire en français », vient suggérer des possibles, 
montrer des ouvertures dans la langue française pour recevoir le parler noir. Lavoie choisit de puiser dans la littérature antillaise afin de décentrer la langue française. C'est grâce aux œuvres des auteurs antillais Patrick Chamoiseau et Jacques Roumain, dont l'écriture fait preuve d'une véritable recherche stylistique et linguistique, qu'elle proposera une solution à la problématique de la traduction du Black English en français. Elle établira les liens littéraires, ainsi que la parenté socio-linguistique et historique, entre le français créolisé et le vernaculaire noir américain, en prenant bien soin de préciser qu'il ne s'agit pas là d'équivalents. Le français créolisé, grâce à un procédé de compensation, assumerait plutôt la même fonction identificatrice au sein du texte que le Black English. Ces pistes esquissées par Lavoie pourraient mener à une entreprise de retraduction qui donnerait enfin à lire l'œuvre de Twain, dans toute sa plurivocalité, au public français, afin de « réactiver à sa pleine mesure la charge profondément revendicatrice qu'elle véhicule». (p. 213). En définitive, cette étude constitue un apport indiscutable à la traductologie, et plus particulièrement à l'étude du sociolecte en traduction. Grâce à l'analyse minutieuse des traductions, l'auteure jette un éclairage nouveau sur l'œuvre de Mark Twain et en fait ressortir toute la richesse plurilinguistique. Au terme de la lecture de cet ouvrage et à la lumière des enjeux soulevés par la traduction française du vernaculaire noir américain, on aurait souhaité lire un extrait traduit par Lavoie mettant en pratique les principes énoncés dans le dernier chapitre, comme elle l'avait fait dans sa thèse de doctorat. Mais le lecteur devra plutôt attendre qu'un traducteur entreprenne cette tâche de retraduction monumentale pour enfin lire un Huckleberry Finn en français à la hauteur de l'original.

\section{Savoyane Henri-Lepage Université McGill}

Sherry Simon and Paul St-Pierre (eds.). Changing the Terms. Translating in the Postcolonial Era, Ottawa, University of Ottawa Press, 2000, 305 p.

Illustration de couverture : l'image tendre et charmante de deux mainates sur les branches d'un arbre, l'un rouge, l'autre bleu, l'un triste, l'autre malicieux. Celui du haut regarde vers l'Ouest, l'autre vers l'Est. Bien trouvé pour un ouvrage traitant du postcolonialisme. La curiosité pousse à chercher en page intérieure 